\title{
Mode-Coupling Theory for Structural and Conformational Dynamics of Polymer Melts
}

\author{
S.-H. Chong ${ }^{1, *}$ and M. Fuchs ${ }^{2}$ \\ ${ }^{1}$ Physik-Department, Technische Universität München, 85747 Garching, Germany \\ ${ }^{2}$ Institut Charles Sadron, 6 rue Boussingault, 67083 Strasbourg, France \\ (Received 23 January 2002; published 22 April 2002)
}

\begin{abstract}
A mode-coupling theory for dense polymeric systems is developed which unifyingly incorporates the segmental cage effect relevant for structural slowing down and polymer chain conformational degrees of freedom. An ideal glass transition of polymer melts is predicted which becomes molecular-weight independent for large molecules. The theory provides a microscopic justification for the use of the Rouse theory in polymer melts, and the results for Rouse-mode correlators and mean-squared displacements are in good agreement with computer simulation results.
\end{abstract}

PACS numbers: 64.70.Pf, 61.20.Lc, 61.25.Hq

Polymeric materials often find applications as amorphous solids or glasses. During their processing, the structural relaxation in polymeric melts plays an important role because it affects transport and viscoelastic properties. An understanding of conformational dynamics is also required for single-chain diffusional processes. Whereas a number of phenomenological concepts have been invented, no microscopic theory exists which successfully explains the slow structural and conformational relaxations of polymeric melts close to the glass transition, as, for example, studied in detailed computer simulations of model systems [1].

We present a first-principles theory for structural and conformational dynamics of unentangled polymer chains using the site formalism which has already provided a good description of their static packing [2]. Building upon the mode-coupling theory (MCT) for simple and lowmolecular systems [3-5], our macromolecular extension captures the segmental "cage effect" which causes a (asymptotically) molecular-weight-independent ideal glass transition driven by steric hindrance on microscopic length scales. A decoupling of local collective structural relaxation from single-chain conformational fluctuations is obtained, leading to a first-principles derivation of the Rouse model [6]. This widely used model assumes a chain to be in a Markovian heat bath, and therefore its applicability requires a time-scale decoupling which our theory achieves. Entanglement effects [6] are neglected in our work because we do not ensure uncrossability of chains and consider only local isotropic forces [7].

A system of $n$ chains, consisting of $N$ identical monomers or segments, distributed with density $\rho$ is considered. In the site formalism [8], the structural variables are the monomer-density fluctuations for wave vector $\vec{q}$, $\rho_{\vec{q}}^{a}=\sum_{i=1}^{n} \exp \left(i \vec{q} \cdot \vec{r}_{i}^{a}\right)$, where $\vec{r}_{i}^{a}$ denotes the position of the $a$ th monomer in the $i$ th chain. Structural dynamics shall be described by coherent density correlators, $F_{q}^{a b}(t)=\left\langle\rho_{\vec{q}}^{a}(t)^{*} \rho_{\vec{q}}^{b}\right\rangle / n$. Here $\langle\cdots\rangle$ denotes canonical averaging for temperature $T$. A single (or tagged) polymer (labeled $s$ ) exhibits density fluctuations $\rho_{\vec{q}, s}^{a}=$ $\exp \left(i \vec{q} \cdot \vec{r}_{s}^{a}\right)$, and correlators, $F_{q, s}^{a b}(t)=\left\langle\rho_{\vec{q}, s}^{a}(t)^{*} \rho_{\vec{q}, s}^{b}\right\rangle$, characterize the single-chain dynamics.

We apply MCT equations in the site representation [5] to flexible macromolecules, expecting them to capture intermolecular caging in polymeric melts. The required inputs are the static structure factors $S_{q}^{a b}=F_{q}^{a b}(0), w_{q}^{a b}=$ $F_{q, s}^{a b}(0)$, and the direct correlation functions $c_{q}^{a b}$ [8]. There are severe difficulties to solve the $(N \times N)$-matrix MCT equations for polymers because the degrees of polymerization $N$ of interest are large. The simplifications adopted here are to neglect chain-end effects for $c_{q}^{a b}$ [2], $c_{q}^{a b}=$ $c_{q}$, and to consider the site-averaged correlator, $F_{q}(t)=$ $(1 / N) \sum_{a, b=1}^{N} F_{q}^{a b}(t)$, which deals with the total monomerdensity fluctuations. This mean-field-like approximation replaces the site-specific surroundings of a segment by an averaged one. It is supported by the observation that $S_{q}=F_{q}(0)$ captures the static correlations on the segmental length scale [2].

We find a set of scalar equations for the normalized coherent correlator $\phi_{q}(t)=F_{q}(t) / S_{q}$ :

$$
\begin{gathered}
\partial_{t}^{2} \phi_{q}(t)+\Omega_{q}^{2} \phi_{q}(t)+ \\
\Omega_{q}^{2} \int_{0}^{t} d t^{\prime} m_{q}\left(t-t^{\prime}\right) \partial_{t^{\prime}} \phi_{q}\left(t^{\prime}\right)=0, \\
m_{q}(t)=\frac{1}{2} \int d \vec{k} V(\vec{q} ; \vec{k}, \vec{p}) \phi_{k}(t) \phi_{p}(t) .
\end{gathered}
$$

Here $\Omega_{q}^{2}=q^{2} v^{2} / S_{q}$ and $V=\rho_{m} S_{q} S_{k} S_{p}\left\{\vec{q} \cdot\left[\vec{k} c_{k}+\right.\right.$ $\left.\left.\vec{p} c_{p}\right]\right\}^{2} /(2 \pi)^{3} q^{4}$ with $v$ denoting the monomer thermal velocity, $\rho_{m}=N \rho$, and $\vec{p}=\vec{q}-\vec{k}$. These equations are formally identical to the ones for simple systems [3]. On the other hand, one finds for the single-chain dynamics:

$$
\begin{gathered}
\partial_{t}^{2} \mathbf{F}_{q, s}(t)+\mathbf{\Omega}_{q, s}^{2} \mathbf{F}_{q, s}(t)+ \\
\mathbf{\Omega}_{q, s}^{2} \int_{0}^{t} d t^{\prime} \mathbf{m}_{q, s}\left(t-t^{\prime}\right) \partial_{t^{\prime}} \mathbf{F}_{q, s}\left(t^{\prime}\right)=\mathbf{0} \\
m_{q, s}^{a b}(t)=\sum_{c} \frac{w_{q}^{a c}}{q^{2}} \int d \vec{k} V_{s}(\vec{q} ; \vec{k}, \vec{p}) F_{k, s}^{c b}(t) \phi_{p}(t) .
\end{gathered}
$$


Here $\boldsymbol{\Omega}_{q, s}^{2}=q^{2} v^{2} \mathbf{w}_{q}^{-1}$ and $V_{s}=\rho_{m}(\vec{q} \cdot \vec{p} / q)^{2} S_{p} c_{p}^{2} /$ $(2 \pi)^{3}$.

A traditional description of the single-chain dynamics is in terms of Rouse modes [6]. For discrete chains, Rouse-mode correlators are defined by $C_{p p^{\prime}}(t)=\left\langle\vec{X}_{p}(t)\right.$. $\left.\vec{X}_{p^{\prime}}\right\rangle / 3 N$ with $\vec{X}_{p}=\sqrt{2 / N} \sum_{a=1}^{N} \vec{r}_{s}^{a} \cos [(a-1 / 2) p \pi /$ $N]$. Since $\rho_{\vec{q}, s}^{a} \approx 1+i \vec{q} \cdot \vec{r}_{s}^{a}$ for small $\vec{q}, C_{p p^{\prime}}(t)$ can be expressed as a linear combination of $F_{q, s}^{a b}(t)$ for $q \rightarrow 0$. Here, it is neither assumed that $C_{p p^{\prime}}(t)$ are diagonal nor assumed that the decay is exponential. Therefore, our microscopic theory can test the validity of the Rouse theory.

The polymers considered here shall be modeled as follows. First, chains are assumed to be Gaussian, for which $w_{q}^{a c}=\exp \left[-q^{2}|a-c| b^{2} / 6\right]$ with the statistical segment length $b$. Second, each monomer is modeled as a hard sphere of diameter $d$ and mass $m$, and we set $b=d$. All equilibrium properties are then specified by the packing fraction $\varphi=\pi \rho_{m} d^{3} / 6$ and the degree of polymerization $N$. Third, $S_{q}$ and $c_{q}$ are evaluated from the polymer reference-interaction-site-model theory [2]. Let us add that the value of $\varphi$ can become larger than 1 for very dense systems since corrections due to nonphysical intrapolymer monomer overlap [9] are not taken into account in this Letter. From here on, the units will be chosen so that $d=v=m=1$.

The derived MCT equations (1) and (2) exhibit an ideal liquid-glass transition upon increasing $\varphi$ at a critical value $\varphi_{c}$ as discussed for simple systems [4,10]. The solid line in Fig. 1 exhibits the resulting glass-form factors $f_{q}^{c}=$ $\phi_{q}(t \rightarrow \infty)$ at $\varphi_{c}$ for $N=10$, and the dashed line exhibits $S_{q}$ multiplied by 0.15 . The $f_{q}^{c}$ also measure the amplitude of the slow structural relaxation in the liquid state. Inspection of Eq. (2) shows that the liquid-glass transition is driven mainly by the changes of $S_{q}$ for $q_{\max } \approx 7$, where $f_{q}^{c}$ is maximal, i.e., by local fluctuations connected to the

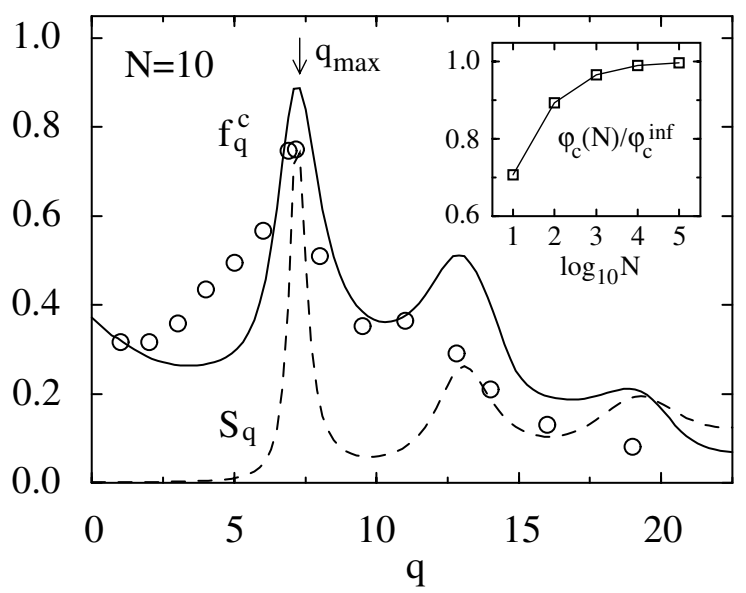

FIG. 1. The solid line exhibits the glass-form factors $f_{q}^{c}$ at $\varphi=\varphi_{c}$ for $N=10$ along with the simulation result (circles) for a similar system taken from Ref. [14]. The dashed line denotes $S_{q}$ multiplied by 0.15 . The inset shows $\varphi_{c}(N)$ rescaled by $\varphi_{c}^{\mathrm{inf}}=\varphi_{c}(N \rightarrow \infty) \approx 1.309$ as a function of $\log _{10} N$. average nearest-neighbor monomer distance (the cage effect). With varying $q, f_{q}^{c}$ oscillates in phase with $S_{q}$ as found in simple systems $[4,10]$. The circles denote the result of molecular-dynamics simulation performed for a similar system [11-14]. The agreement is semiquantitative especially for $q \approx q_{\max }$, which is the relevant $q$ range for the ideal glass transition [15]. The inset demonstrates that $\varphi_{c}(N)$ becomes independent of $N$ for large $N$. This is because the glassy arrest is driven by the local cage effect, and the global chain size plays a gradually smaller role for larger $N$ [16].

For packing fractions $\varphi$ close to but below $\varphi_{c}$, correlator $\phi_{A}(t)$ of any variable $A$ coupling to density fluctuations exhibits slow structural relaxation and decays in two steps: the decay towards the plateau $f_{A}^{c}$, followed by the decay to zero (so-called $\alpha$ decay) [3,4]. Detailed analysis of $\phi_{q}(t)$ and $F_{q, s}^{a b}(t)$ will be presented in later publications. Here, only the Rouse-mode correlators $C_{p p^{\prime}}(t)$ shall be studied. Typically, their off-diagonal elements are found to be much smaller (at most only a few percent) compared to the diagonal ones at all times. Therefore, the normalized diagonal elements, $c_{p}(t)=C_{p p}(t) / C_{p p}(0)$, shall be considered. Figure 2 exhibits representative results for $N=10$ for a reduced packing fraction $\left(\varphi-\varphi_{c}\right) / \varphi_{c}=-10^{-2}$.

The curves shown in Fig. 2 do not clearly exhibit the two-step relaxation scenario. This is because the plateaus of $c_{p}(t)$ are so large, $f_{p}^{c}>0.9$, that only less than $10 \%$ of the decay is left for the relaxation towards the plateau. Thus, most of the relaxation of $c_{p}(t)$ occurs in the $\alpha$ regime. Let us characterize the $\alpha$ time scale by $c_{p}\left(\tau_{p}\right)=f_{p}^{c} / 20$. The corresponding $\alpha$ time scale $\tau_{q \max }$ shall be introduced for the coherent correlator $\phi_{q}(t)$

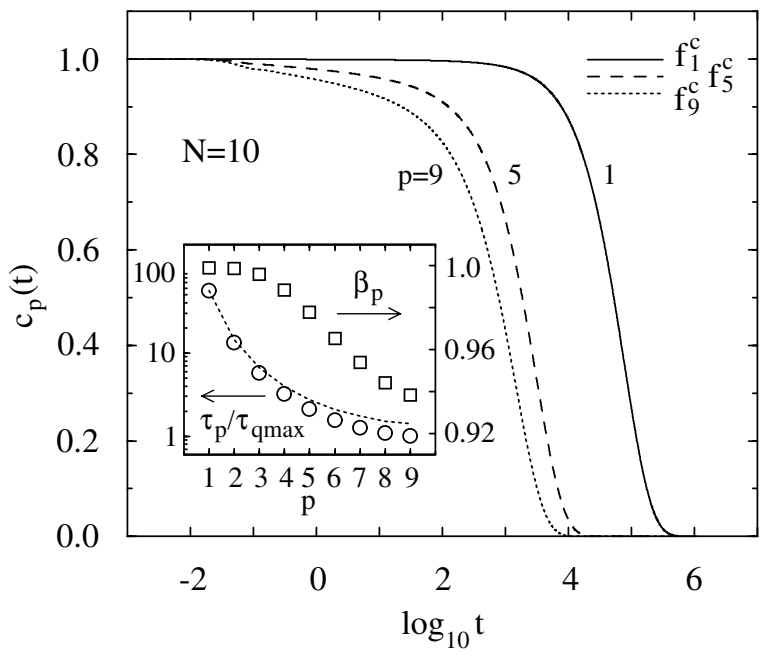

FIG. 2. Rouse-mode correlators $c_{p}(t)$ of $p=1$ (solid line), $p=5$ (dashed line), and $p=9$ (dotted line) for $N=10$ and $\left(\varphi-\varphi_{c}\right) / \varphi_{c}=-10^{-2}$. The horizontal lines mark the plateaus $f_{p}^{c}$. The inset shows the ratio $\tau_{p} / \tau_{q \text { max }}$ of the $\alpha$-relaxation times (circles) and the stretching exponent $\beta_{p}$ (squares) as a function of $p$. The dotted line denotes a fitting based on the formula $\tau_{p}=\zeta / 12 \sin ^{2}(p \pi / 2 N)$. 
for $q=q_{\max }$, characterizing the local dynamics of the surrounding medium. The ratio $\tau_{p} / \tau_{q \max }$ is shown in the inset as a function of $p$. For small $p$, the scales $\tau_{p}$ are much larger than the local scale $\tau_{q \max }$.

The Rouse theory assumes that all dynamical correlations in the surroundings are much faster than the singlechain dynamics [6]. Since a polymer is surrounded by identical polymers, the assumption of the time-scale separation cannot be justified a priori. Our microscopic theory verifies this central assumption. The Rouse theory predicts, within our units, $\tau_{p}=\zeta / 12 \sin ^{2}(p \pi / 2 N)$, where $\zeta$ denotes the monomer friction. This formula, using $\zeta$ as a fit parameter, is shown as the dotted line, and we found $\zeta / \tau_{q \max }=16.5$.

The shape of $c_{p}(t)$ in the $\alpha$ regime is often characterized by the stretching exponent $\beta_{p}$ of the Kohlrausch-law fit: $c_{p}(t) \propto \exp \left[-\left(t / \tau_{p}^{\prime}\right)^{\beta_{p}}\right]$. We found $\beta_{p}>0.9$ for all $p$, as shown in the inset. For small $p, \beta_{p}$ is close to 1 due to the large separation of the scales $\tau_{p}$ and $\tau_{q \max }$, as discussed above. $\beta_{p}$ decreases as $p$ increases since for large $p$ the scales $\tau_{p}$ become comparable to $\tau_{q \max }$. However, $\beta_{p}$ remains close to 1 even for large $p$. This is because the plateaus $f_{p}^{c}$ are high, as explained in Ref. [17]. Thus, all our $c_{p}(t)$ exhibit nearly Debye relaxation as assumed by the Rouse theory. Let us note that the found features for $c_{p}(t)$ hold also for $N=100$, and are in agreement with simulation results [12].

Of interest are the monomer mean-squared displacement (MSD) $g_{a}(t)=\left\langle\left[\vec{r}_{s}^{a}(t)-\vec{r}_{s}^{a}(0)\right]^{2}\right\rangle$ and the center-of-mass MSD, $g_{C}(t)$. Let us also introduce the monomeraveraged MSD, $g_{M}(t)=(1 / N) \sum_{a=1}^{N} g_{a}(t)$. Figure 3 exhibits $g_{M}(t)$ and $g_{C}(t)$ for $N=10$ and 100 for $\left(\varphi-\varphi_{c}\right) /$ $\varphi_{c}=-10^{-2}$. For short times, the MSD exhibits ballistic motion: $g_{X}(t) \propto t^{2}(X=M$ or $C)$. As the time increases, the MSD begins to be suppressed due to the cage effect and there appears the so-called $\beta$ regime, where $g_{X}(t)$ is close to the square of the critical localization length, $g_{X}(t) \approx 6\left(r_{X}^{c}\right)^{2}$. For this regime, there holds $[3,4,18]$

$$
g_{X}(t)=6\left(r_{X}^{c}\right)^{2}-6 h_{X} G(t), \quad X=M, C,
$$

where $G(t)$ denotes the $\beta$ correlator and $h_{X}$ denotes the critical amplitude. Thus, $g_{M}(t)$ and $g_{C}(t)$ cross their plateaus $6\left(r_{X}^{c}\right)^{2}$ at the same time. The value $r_{M}^{c} \approx 0.077$, quantifying the monomer localization, for both $N=10$ and 100 is consistent with Lindemann's melting criterion. $r_{C}^{c}$ is reduced by about $1 / \sqrt{N}$ compared to $r_{M}^{c}$, as expected for independent motions of constituent monomers. The asymptotic law (5) for each MSD is drawn as a dashed line, and its range of validity is indicated by diamonds. The trends in $g_{X}(t)$ up to the $\beta$ regime are qualitatively similar to the ones for a sphere in a simple system [18].

The increase of $g_{X}(t)$ above the plateau $6\left(r_{X}^{c}\right)^{2}$ towards the diffusion asymptote, $g_{X}(t) \approx 6 D t$ with the diffusivity $D$, is the $\alpha$ process of the MSD. In contrast to $g_{C}(t), g_{M}(t)$ in this regime is significantly affected by chain connectivity since the segments participate in the conformational
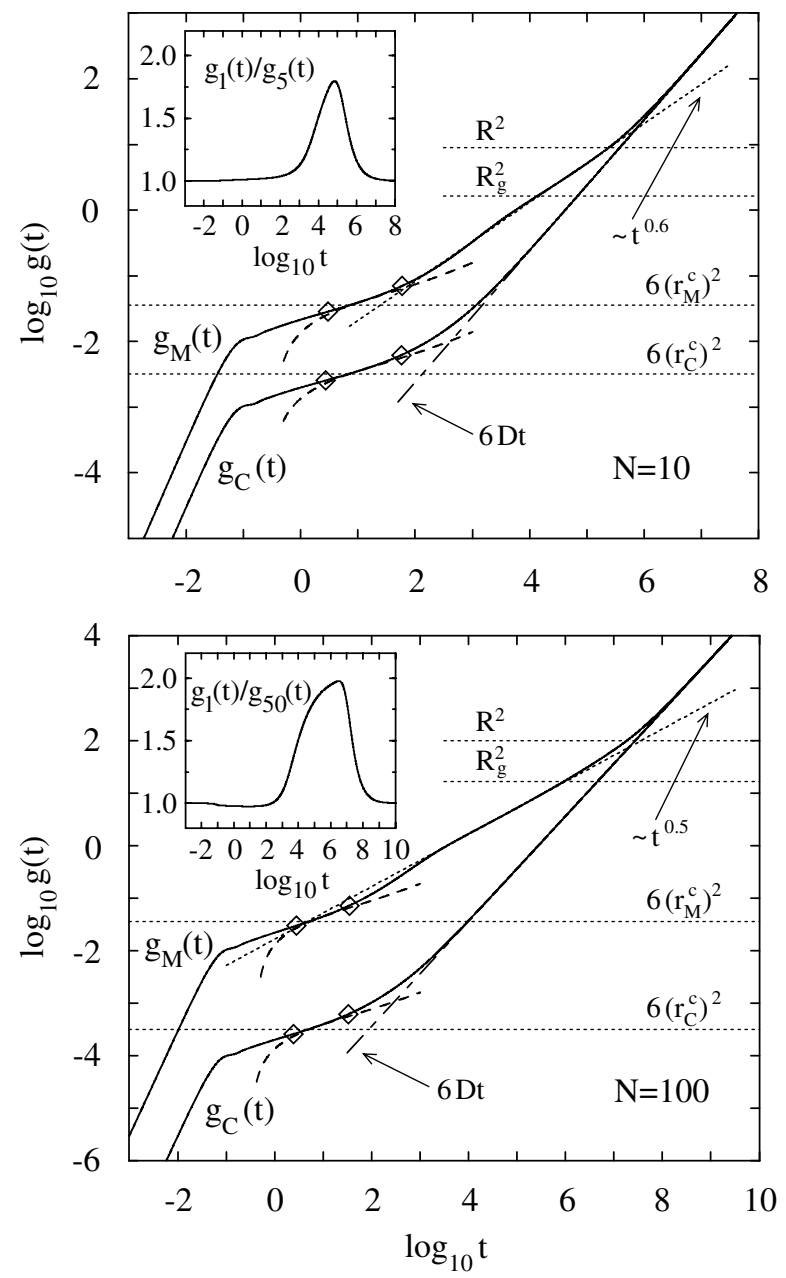

FIG. 3. Double logarithmic presentation of the MSDs $g_{M}(t)$ and $g_{C}(t)$ (solid lines) for $N=10$ (upper panel) and 100 (lower panel) for $\left(\varphi-\varphi_{c}\right) / \varphi_{c}=-10^{-2}$. The dashed lines denote the asymptotic formula given in Eq. (5). The open diamonds mark the points where the dashed lines differ from the solid ones by $10 \%$. The dash-dotted lines exhibit the diffusion law, 6Dt. The dotted lines show the power laws, $\sim t^{x}$, with $x$ specified in each panel. The horizontal dotted lines from bottom to top successively mark the plateau $6\left(r_{C}^{c}\right)^{2}$ for $g_{C}(t)$, the plateau $6\left(r_{M}^{c}\right)^{2}$ for $g_{M}(t)$, the radius of gyration $R_{g}^{2}$, and the end-to-end distance $R^{2}$. The insets exhibit the ratio $g_{1}(t) / g_{N / 2}(t)$.

motion and most of the relaxation of $c_{p}(t)$ occurs here, as explained above. As a result, there appears a subdiffusive $\left(\sim t^{x}\right)$ regime in $g_{M}(t)$. For $N=10$, the exponent $x$ is 0.60 , which is close to the value (0.63) found in the mentioned simulations [12]. For $N=100$, we find $x=0.5$, as predicted by the Rouse model and by the asymptotic evaluation of our theory. The different value for $N=10$ can be attributed to finite $N$ effects. Thus, we find a strong polymer-specific effect for the beginning of the $\alpha$ process, while no such effect is reflected in $g_{M}(t)$ up to the $\beta$ process.

The insets of Fig. 3 show the ratio of the MSD for end and central monomers, $g_{1}(t) / g_{N / 2}(t)$. The ratio starts from 
1 in the ballistic regime, exhibits a maximum for intermediate times, and tends to 1 in the diffusion regime. It is seen that the ratio remains close to 1 also within the $\beta$ regime. This is because the dynamics here does not reflect the chain connectivity, as explained above. The Rouse theory predicts the maximum to be 2 within the time regime where the monomer MSD exhibits the $t^{1 / 2}$ law, i.e., in the $\alpha$ regime. The result for $N=100$ clearly indicates this behavior, while the maximum for $N=10$ is slightly smaller (1.8) due to finite $N$ effects. The shape of the ratio for $N=10$ in the $\beta$ and $\alpha$ regimes is in semiquantitative agreement with the mentioned simulation results [19]. Thus, the chain-end effect is properly taken into account in our theory even though neglecting that effect for $c_{q}^{a b}$. This is because the matrix structure of Eqs. (3) and (4) is preserved for describing the single-polymer dynamics.

In summary, a first-principles theory for structural slowing down of dense polymer systems has been presented which also provides a microscopic derivation of the Rouse model for unentangled chain melts. Chain connectivity is seen to cause polymer-specific long-time anomalies of the $\alpha$ process. We use the concept of an ideal MCT glass transition, familiar for simple liquids and colloidal suspensions [20], and our results agree well with simulation studies of simple (coarse-grained) polymer models. Our theory thus provides insights into dynamical aspects typical for polymer melts which are also observed in models [21,22] with a more realistic local chemistry.

We thank J. Baschnagel, M. Aichele, and W. Götze for discussions. M.F. was supported by the Deutsche Forschungsgemeinschaft, Grant No. Fu 309/3.

*Present address: Dipartimento di Fisica, INFM and INFM Center for Statistical Mechanics and Complexity, Università di Roma "La Sapienza," Piazzale Aldo Moro 2, I-00185, Roma, Italy.

[1] K. Binder, J. Baschnagel, and W. Paul, Prog. Polym. Sci. (to be published).
[2] K. S. Schweizer and J. G. Curro, Adv. Chem. Phys. 98, 1 (1997).

[3] W. Götze, in Liquids, Freezing and Glass Transition, edited by J.-P. Hansen, D. Levesque, and J. Zinn-Justin (NorthHolland, Amsterdam, 1991), p. 287.

[4] W. Götze and L. Sjögren, Rep. Prog. Phys. 55, 241 (1992).

[5] S.-H. Chong and W. Götze, Phys. Rev. E 65, 041503 (2002).

[6] M. Doi and S. F. Edwards, The Theory of Polymer Dynamics (Oxford University, Oxford, 1986).

[7] K. S. Schweizer, J. Chem. Phys. 91, 5802 (1989); 91, 5822 (1989).

[8] D. Chandler and H. C. Andersen, J. Chem. Phys. 57, 1930 (1972).

[9] K. S. Schweizer and J. G. Curro, Macromolecules 21, 3070 (1988)

[10] T. Franosch, M. Fuchs, W. Götze, M. R. Mayr, and A. P. Singh, Phys. Rev. E 55, 7153 (1997).

[11] C. Bennemann, W. Paul, K. Binder, and B. Dünweg, Phys. Rev. E 57, 843 (1998).

[12] C. Bennemann, J. Baschnagel, W. Paul, and K. Binder, Comput. Theor. Polym. Sci. 9, 217 (1999).

[13] C. Bennemann, J. Baschnagel, and W. Paul, Eur. Phys. J. B 10, 323 (1999).

[14] M. Aichele and J. Baschnagel, Eur. Phys. J E 5, 229 (2001); 5, 245 (2001).

[15] The disagreement for $q<q_{\max }$ may be due to the fact that center-of-mass density fluctuations are not our explicit dynamical variables [14], while the one for $q>q_{\max }$ may be due to a softer Lennard-Jones potential which is employed in the simulation.

[16] G. B. McKenna, in Comprehensive Polymer Science, edited by C. Booth and C. Price (Pergamon, New York, 1989), Vol. 2, p. 311.

[17] W. Götze, A. P. Singh, and Th. Voightmann, Phys. Rev. E 61, 6934 (2000).

[18] M. Fuchs, W. Götze, and M. R. Mayr, Phys. Rev. E 58, 3384 (1998).

[19] J. Baschnagel (private communication).

[20] W. Götze, J. Phys. Condens. Matter 11, A1 (1999).

[21] A. van Zon and S. W. de Leeuw, Phys. Rev. E 58, R4100 (1998).

[22] A. van Zon and S. W. de Leeuw, Phys. Rev. E 60, 6942 (1999). 